\title{
Open-Loop Power Control Error in Cellular CDMA Overlay Systems
}

\author{
Jiangzhou Wang, Senior Member, IEEE, and Ai Yu
}

\begin{abstract}
Adaptive power control has widely been used in DS/CDMA systems to overcome the so-called "near-far" problem. This paper studies the adaptive open-loop power control of a cellular CDMA system, which is overlaid in the downlink by a narrowband signal. The effects of downlink power allocation schemes to power control error in the presence of narrowband interference are analyzed. In order to get a minimum power control error in the CDMA overlay situations, an optimum downlink power allocation scheme is used, which works well for a wide range of signal to narrowband interference ratio.
\end{abstract}

Index Terms-CDMA, downlink power allocation, narrowband interference suppression, open-loop power control.

\section{INTRODUCTION}

A S WELL KNOWN, a direct sequence (DS) code division multiple access (CDMA) system is susceptible to near-far interference, which occurs when the base station input includes one or more other CDMA signals that are stronger than the desired signal. The near-far effect can be reduced by adjusting the transmitted power of all mobile users so that the base station gets the same power from the received signal of each transmission. Two types of power control are often considered: closed-loop power control and open-loop power control. In a closed-loop power control, according to the received signal power at a base station, the base station sends a command to a mobile to adjust the transmit power of the mobile. However, in an open-loop power control, a mobile user adjusts its transmit power according to its received power in downlink. In this paper, an adaptive open-loop power control algorithm [1] is adopted. The algorithm produces an estimate of the received power at the mobile by averaging squared outputs of the correlator.

The open-loop power control error usually results from the factors such as the accuracy of power measurement at a mobile, the dynamic ranges of the transmit power of mobiles and the loop delay. In this paper, the measurement error in the presence of narrowband interference (NBI) is studied. It has been shown in [2] that it may be possible for a mobile to do real-time power measurements in which the power control error is lognormally distributed with standard deviation of three $\mathrm{dB}$ or less, in the absence of NBI.

Because the open-loop power control algorithm uses the downlink received power to determine the uplink transmit power, the downlink transmit power allocation should be

Manuscript received February 10, 2000; revised February 14, 2001. The authors are with the Department of Electrical and Electronic Engineering, The University of Hong Kong, Hong Kong (e-mail: jwang@eee.hku.hk). Publisher Item Identifier S 0733-8716(01)04707-2.

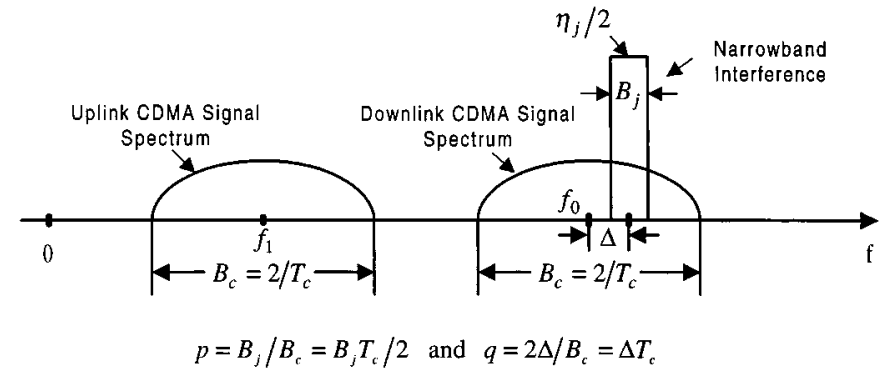

Fig. 1. Power spectrum of the uplink and downlink CDMA signals and NBI.

considered. As known, downlink power control (power allocation) has been used in cellular CDMA systems to reduce the adjacent-cell interference and to let all the users of the cell of interest have the same $C / I$ to achieve high capacity [3], [4]. For different downlink power control algorithms, the mobiles will be allocated different power, which will result in different power control error in the uplink.

A CDMA system is often assumed to share common spectrum with narrowband microwave users [5], [6]. Because of the presence of the narrowband waveforms, interference suppression filters are employed to reject the NBI. So far, spectrum overlay on uplink with perfect power control has been assumed in the literature. This paper studies the effects of power control error on performance of the CDMA overlay system, which is overlaid on downlink by a narrowband signal (as shown in Fig. 1).

The paper is organized as follows. The downlink system model is given in Section II. In Section III, the open-loop power control in the presence of NBI is studied. Numerical results are given in Section IV. The conclusion will be presented in the last section.

\section{DOWNLINK SySTEM DESCRIPTION}

Neglecting the white noise on the downlink, the waveforms received by each mobile is composed of the desired signal, a number of interfering signals from the base station of the cell of interest and the adjacent-cell base stations, and the NBI.

Assuming that all spreading codes of each cell are orthogonal and that the downlink transmissions of a cell are synchronized, the intracell interference can be ignored because of the same transmission delay. However, the interfering signals from adjacent-cell base stations cannot be assumed to be synchronized with the desired signal because of different transmission delays. This interference becomes significant when a mobile moves toward the boundary of its cell. 


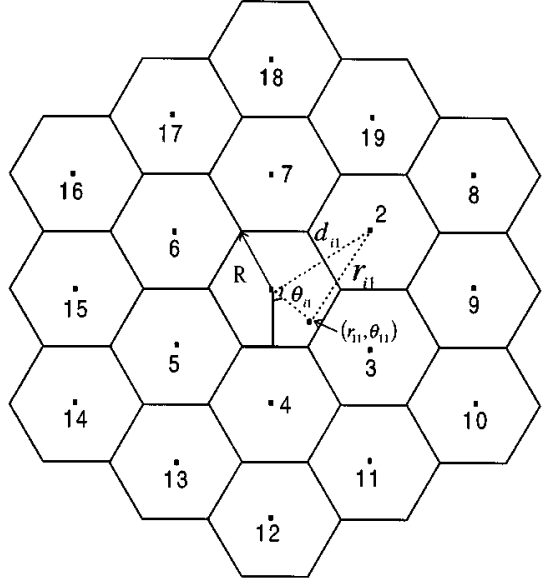

Fig. 2. Downlink adjacent cell interference geometry.

In order to study the downlink adjacent-cell interference, two layers of cells are considered (see Fig. 2). Each user is assumed to be located independently of all the other users and uniformly distributed over the area of the cell. The location of a reference user in the first cell (the cell of interest) is $\left(r_{11}, \theta_{11}\right)$ (see Fig. 2), where $r_{11}$ and $\theta_{11}$ stand for the distance and angle of the mobile from its base station. The distance between the base station of the $i$ th adjacent cell and the reference mobile of the first cell is given by

$$
r_{i 1}=\sqrt{d_{i 1}^{2}+r_{11}^{2}-2 d_{i 1} r_{11} \cos \theta_{i 1}}, \quad i=2,3, \ldots, 19
$$

where $d_{i 1}$ is the distance between the first base station and the $i$ th base station, and

$$
d_{i 1}= \begin{cases}\sqrt{3} R, & i=2,3, \ldots, 7 \\ 2 \sqrt{3} R, & i=8,10,12,14,16,18 \\ 3 R, & i=9,11,13,15,17,19\end{cases}
$$

where $R$ stands for the radius of the hexagonal cell. In (1), $\theta_{i 1}$ is the angle between $d_{i 1}$ and $r_{11}$. The relationship between $\theta_{i 1}$ and $\theta_{11}$ for various values of $i$ is given by

$$
\cos \left(\theta_{i 1}\right)=\left\{\begin{array}{ll}
\cos \left[\theta_{11}+(i+2) \pi / 3\right], & 2 \leq i \leq 7 \\
\cos \left(\theta_{11}+i \pi / 6\right), & 8 \leq i \leq 19
\end{array} .\right.
$$

The channel is modeled as a frequency nonselective channel with Rayleigh statistics and lognormal distributed shadowing. The mobile receiver is a conventional coherent receiver with a Wiener suppression filter at its front end [5]. Assuming that there are $K$ active users for each cell, the received signal at mobile user 1 (reference user) can be written as

$$
\begin{aligned}
r\left(\left.t\right|_{r_{11}, \theta_{11}}\right)= & \sum_{i=1}^{19} \sum_{k=1}^{K} \sqrt{2 P_{R} \xi_{i k} \cdot r_{i 1}^{-\gamma}} a_{i}(t) e^{\zeta_{i}(t)} c_{i k}\left(t-\tau_{i}\right) \\
& \times b_{i k}\left(t-\tau_{i}\right) \cos \left(2 \pi f_{0} t-\phi_{i}\right)+j(t)
\end{aligned}
$$

where $P_{R}$ is the transmitted power of the base station when the reference user is located at a cell vertex $\left(r_{11}=R\right)$, and $\xi_{i k}(0<$ $\xi_{i k} \leq 1$ ) is the downlink link power adjustment factor (or power control function) and $\xi_{11}=1$ when $r_{11}=R \cdot r_{i 1}^{-\gamma}$ represents the propagation path loss from the $i$ th base station to the reference user and $\gamma$ may take values between two and four. $a_{i}(t)$ is the Rayleigh distributed random variable with unitary power, i.e., $E\left\lfloor a_{i}^{2}(t)\right\rfloor=1 \cdot e^{\zeta_{i}(t)}$ is the log-normal shadowing with random variable $\zeta_{i}(t)$, taking zero mean and a typical variance of eight $\mathrm{dB}$. The single subscript of $a_{i}(t)$ and $\zeta_{i}(t)$ means that all the signals transmitted by each base station propagate on the same path, and undergo the same fading and shadowing. $c_{i k}(t)$ and $b_{i k}(t)$ represent the spreading sequence with chip duration $T_{c}$ and the binary data sequence with duration $T$ of user $k$ of cell $i$, respectively. $\tau_{i}$ and $\phi_{i}$ are the corresponding time delay and phase, respectively. Note that $\tau_{i}$ and $\phi_{i}$ are the same for all users of cell $i$, because of the synchronous downlink transmission. $f_{0}$ is the CDMA carrier frequency on downlink. $j(t)$ represents the NBI, which is modeled as a zero-mean Gaussian random process with a double-sided power spectral density of $\eta_{j} / 2$ over the bandwidth of $B_{j}$ (see Fig. 1), and is given by

$$
j(t)=j_{c}(t) \cos \left[2 \pi\left(f_{0}+\Delta\right) t\right]-j_{s}(t) \sin \left[2 \pi\left(f_{0}+\Delta\right) t\right] .
$$

The power of $j(t)$ is given by

$$
P_{j}=E\left\lfloor j^{2}(t)\right\rfloor=E\left\lfloor j_{c}^{2}(t)\right\rfloor=E\left\lfloor j_{s}^{2}(t)\right\rfloor=\eta_{j} B_{j} .
$$

Notice that for simple analysis, Gaussian model is assumed for NBI. However, the analytical results in this paper can be extended to other models of NBI, since the bandwidth, frequency offset, and power of the NBI signal are mainly involved in final analytical results. In microcellular models, the cell size is very small, compared to conventional cellular models. Therefore, the microwave NBI power can be assumed to be the same within a cell. Since $j(t)$ is Gaussian with bandwidth $B_{j}$ and flat spectrum $\eta_{j} / 2$, the autocorrelation function of $j(t)$ is given by

$$
\begin{aligned}
R_{j}(\tau) & =E[j(t) j(t+\tau)] \\
& =P_{j} \operatorname{sinc}\left(\pi B_{j} \tau\right) \cos \left[2 \pi\left(f_{0}+\Delta\right) \tau\right] \\
& =P_{j} \operatorname{sinc}\left(2 \pi p \tau / T_{c}\right) \cos \left(2 \pi f_{0} \tau+2 \pi q \tau / T_{c}\right)
\end{aligned}
$$

where $\operatorname{sinc}(x)=\sin (x) / x, p=B_{j} T_{c} / 2$ is defined as the ratio of the interference bandwidth to the spread spectrum bandwidth, $q=\Delta T_{c}$ is defined as the ratio of the offset $(\Delta)$ of the interference carrier frequency to the half $\left(1 / T_{c}\right)$ of the spread spectrum bandwidth.

The suppression filter is assumed to be a double-sided Wiener filter with $M$ taps on each side. The number of total taps is $2 M+1$. Its impulse response is $\sum_{m=-M}^{M} \alpha_{m} \delta\left(t-m T_{c}\right)$, where $\alpha_{0}=1$ and $\alpha_{m}=\alpha_{-m}$. Therefore, the signal at the output of the suppression filter is given by

$$
r_{f}\left(\left.t\right|_{r_{11}, \theta_{11}}\right)=\sum_{m=-M}^{M} \alpha_{m} r\left(t-\left.m T_{c}\right|_{r_{11}, \theta_{11}}\right) .
$$

Assuming $\tau_{1}=\phi_{1}=0$ for the first cell, the output of despreader/demodulator at time $l T$, is given by

$$
\begin{aligned}
& Z\left(\left.l T\right|_{r_{11}, \theta_{11}}\right) \\
& \quad=\frac{1}{T} \int_{(l-1) T}^{l T} r_{f}\left(\left.t\right|_{r_{11}, \theta_{11}}\right) \cdot 2 c_{11}(t) \cos \left(2 \pi f_{0} t\right) d t
\end{aligned}
$$


where $2 \cos \left(2 \pi f_{0} t\right)$ and $c_{11}(t)$ are the recovered carrier and the spreading sequence of the reference user. Since high frequency terms are removed by the lowpass filter following the mixer of the demodulator, the above expression reduces to

$$
\begin{aligned}
Z\left(\left.l T\right|_{r_{11}, \theta_{11}}\right) \approx & \sqrt{2 P_{R} \xi_{11} r_{11}^{-\gamma}} a_{1}(l T) e^{\xi_{1}(l T)} b_{11}(l T) \\
& +J(l T)+I(l T) .
\end{aligned}
$$

Note that, in the following, " $l T$ " is replaced by " $l$ " for simple notation. The first term on the right hand side of (10) is the desired component, corresponding to the zeroth tap of the suppression filter. However, the contribution of the desired signal through the nonzeroth taps is very minor, compared to the first term, and, thus, is neglected like [5]. The average desired signal power is

$$
S=2 P_{R} \xi_{11} r_{11}^{-\gamma} e^{2 \sigma_{\zeta_{1}}^{2}}
$$

The second term on the right hand side of (10) is the NBI component and is given by

$$
\begin{aligned}
J(l)= & \frac{1}{T} \sum_{m=-M}^{M} \alpha_{m} \int_{(l-1) T}^{l T} j\left(t-m T_{c}\right) 2 c_{11}(t) \\
& \times \cos \left(2 \pi f_{0} t\right) d t .
\end{aligned}
$$

The variance of the NBI is derived in the Appendix A and is given by

$$
\begin{aligned}
\sigma_{J}^{2}= & \frac{P_{j}}{N} \sum_{m_{1}=-M}^{M} \alpha_{m_{1}} \\
& \times \sum_{m_{2}=-M}^{M} \alpha_{m_{2}}\left[Q\left(m_{1}, m_{2}\right)+Q\left(-m_{1},-m_{2}\right)\right] \\
= & \frac{P_{j}}{N} \hat{\sigma}_{J}^{2}
\end{aligned}
$$

where

$$
\begin{aligned}
\hat{\sigma}_{J}^{2}= & \sum_{m_{1}=-M}^{M} \alpha_{m_{1}} \\
& \times \sum_{m_{2}=-M}^{M} \alpha_{m_{2}}\left[Q\left(m_{1}, m_{2}\right)+Q\left(-m_{1},-m_{2}\right)\right]
\end{aligned}
$$

and $Q\left(m_{1}, m_{2}\right)$ is given by (A7).

The third term on the right-hand side of (10) is the adjacent-cell interference, and is expressed as

$$
I(l)=\sum_{i=2}^{19} a_{i}(l) e^{\zeta_{i}(l)} \sum_{k=1}^{K} \sqrt{2 P_{R} \xi_{i k} r_{i 1}^{-\gamma}} I_{i, 1}(l) \cos \phi_{i}
$$

where

$$
I_{i, 1}(l)=\frac{1}{T} \int_{(l-1) T}^{l T} b_{i k}\left(t-\tau_{i}\right) c_{i k}\left(t-\tau_{i}\right) c_{11}(t) d t .
$$

When $K$ is large, $I_{i, 1}(l)$ can be approximated by a Gaussian random variable with the variance of $\operatorname{var}\left\lfloor I_{i, 1}(l)\right\rfloor=2 /(3 N)$ [7]. Then, the variance of $I(l)$ is given by

$$
\sigma_{I}^{2}=\frac{2 K}{3 N} e^{2 \sigma_{\zeta_{i}}^{2}} \sum_{i=2}^{19} P_{R} E\left[\xi_{i k}\right] r_{i 1}^{-\gamma}
$$

where $E\left[\xi_{i k}\right]$ stands for the mean of downlink power adjustment factor, $\xi_{i k}$, and is discussed in the following.

\section{- Without Downlink Power Control}

If there is no downlink power control (i.e., $\xi_{i k}=1$ ), the transmit power at a base station is the same for all users of the ith cell. Thus

$$
E\left[\xi_{i k}\right]=1 .
$$

\section{- With Optimum Downlink Power Control}

The criterion for optimum downlink power control is that the output signal to interference ratio (SIR) of a mobile receiver is the same wherever the mobile user is. In the presence of NBI, the total downlink interference should include the adjacent-cell interference and NBI. Therefore, the SIR at the output of the despreader of the mobile receiver is

$$
\begin{aligned}
\operatorname{SIR}\left(r_{11}, \theta_{11}\right) & =\frac{S}{\sigma_{I}^{2}+\sigma_{J}^{2}} \\
& =\frac{2 P_{R} \xi_{11} r_{11}^{-\gamma} e^{2 \sigma_{\zeta 1}^{2}}}{\frac{2 K P_{R}}{3 N} E\left[\xi_{i k}\right] e^{2 \sigma_{\zeta 1}^{2}} \sum_{i=2}^{19} r_{i 1}^{-\gamma}+\sigma_{J}^{2}} \\
& =\frac{P_{S} \xi_{11}\left(r_{11} / R\right)^{-\gamma}}{\frac{K P_{s}}{3 N} E\left[\xi_{i k}\right] \sum_{i=2}^{19}\left(r_{i 1} / R\right)^{-\gamma}+\frac{P_{j}}{N} \hat{\sigma}_{J}^{2}}
\end{aligned}
$$

where $r_{i 1}$ is given by (1), $P_{s}$ is defined as the average received power at the cell vertices,

$$
\begin{aligned}
P_{s} & =E\left[\left(\sqrt{2 P_{R} R^{-\gamma}} a(t) e^{\zeta(t)}\right)^{2}\right] \\
& =2 P_{R} R^{-\gamma} e^{2 \sigma_{\zeta}^{2}} .
\end{aligned}
$$

When the mobile is located at one of the cell vertices (i.e., $r_{11}=R, \theta_{11}=\pi / 6$ and $\left.\xi_{11}=1\right),(19)$ reduces to

$$
\begin{aligned}
& \operatorname{SIR}(R, \pi / 6) \\
& \quad=\frac{P_{s}}{\left.\frac{K P_{s}}{3 N} E\left[\xi_{i k}\right] \sum_{i=2}^{19}\left(r_{i 1} / R\right)^{-\gamma}\right|_{\left(r_{11}=R, \theta_{11}=\pi / 6\right)}+\frac{P_{J}}{N} \hat{\sigma}_{J}^{2}} .
\end{aligned}
$$

For the same SIR, (19) and (21) should be equal, i.e.,

$$
\operatorname{SIR}\left(r_{11}, \theta_{11}\right)=\operatorname{SIR}(R, \pi / 6) .
$$


TABLE I

$E\left[\xi_{i k}\right]$ WITH $K=20, N=1000, p=0.2, q=0.2, M=4$

\begin{tabular}{l|c|c|c|c}
\hline & $P_{j} / P_{s}=-\infty d B$ & $P_{j} / P_{s}=10 d B$ & $P_{j} / P_{s}=15 d B$ & $P_{j} / P_{s}=20 d B$ \\
\hline$\gamma=2$ & 0.4689 & 0.4801 & 0.4858 & 0.4912 \\
\hline$\gamma=3$ & 0.3675 & 0.3836 & 0.3897 & 0.3936 \\
\hline$\gamma=4$ & 0.3189 & 0.3279 & 0.3310 & 0.3321 \\
\hline
\end{tabular}

From (19), (21) and (22), one obtains

$$
\begin{aligned}
& \frac{P_{s} \xi_{11}\left(r_{11} / R\right)^{-\gamma}}{\frac{K P_{s}}{3 N} E\left[\xi_{i k}\right] \sum_{i=2}^{19}\left(r_{i 1} / R\right)^{-\gamma}+\frac{P_{j}}{N} \hat{\sigma}_{J}^{2}} \\
& =\frac{P_{s}}{\left.\frac{K P_{s}}{3 N} E\left[\xi_{i k}\right] \sum_{i=2}^{19}\left(r_{i 1} / R\right)^{-\gamma}\right|_{\left(r_{11}=R, \theta_{11}=\pi / 6\right)}+\frac{P_{j}}{N} \hat{\sigma}_{J}^{2}} .
\end{aligned}
$$

From (23), the power adjustment factor $\xi_{11}$ is derived

$$
\begin{aligned}
\xi_{11}= & \frac{\left(r_{11} / R\right)^{\gamma}\left[\frac{K P_{s}}{3 N} E\left[\xi_{i k}\right] \sum_{i=2}^{19}\left(r_{i 1} / R\right)^{-\gamma}+\frac{P_{j}}{N} \hat{\sigma}_{J}^{2}\right]}{\left.\frac{K P_{s}}{3 N} E\left[\xi_{i k}\right] \sum_{i=2}^{19}\left(r_{i 1} / R\right)^{-\gamma}\right|_{\left(r_{11}=R, \theta_{11}=\pi / 6\right)}+\frac{P_{j}}{N} \hat{\sigma}_{J}^{2}} \\
= & \frac{\frac{K}{3} E\left[\xi_{i k}\right] \sum_{i=2}^{19}\left(r_{i 1} / r_{11}\right)^{-\gamma}+\frac{P_{j}}{P_{s}} \hat{\sigma}_{J}^{2}\left(r_{11} / R\right)^{\gamma}}{\left.\frac{K}{3} E\left[\xi_{i k}\right] \sum_{i=2}^{19}\left(r_{i 1} / R\right)^{-\gamma}\right|_{\left(r_{11}=R, \theta_{11}=\pi / 6\right)}+\frac{P_{j}}{P_{s}} \hat{\sigma}_{J}^{2}} .
\end{aligned}
$$

In a special case, when $P_{j} / P_{s}$ is very small (i.e., the NBI is absent, $\left.P_{j} / P_{s}=0\right)$, (24) reduces to

$$
\xi_{11} \approx \frac{\sum_{i=2}^{19}\left(r_{i 1} / r_{11}\right)^{-\gamma}}{\left.\sum_{i=2}^{19}\left(r_{i 1} / R\right)^{-\gamma}\right|_{\left(r_{11}=R, \theta_{11}=\pi / 6\right)}},
$$

which becomes the case discussed in [4].

Assuming that the position of each user is uniformly distributed in a cell, i.e.,

$$
f\left(r_{11}, \theta_{11}\right)=\frac{1}{\pi R^{2}}, \quad 0 \leq r_{11} \leq R, \quad 0 \leq \theta_{11} \leq 2 \pi
$$

the expectation of $\xi_{i k}$ is obtained by double integration of (24),

$$
E\left[\xi_{i k}\right]=E\left[\xi_{11}\right]=\frac{1}{\pi R^{2}} \int_{0}^{2 \pi} \int_{0}^{R} \xi_{11} r_{11} d r_{11} d \theta_{11}
$$

By numerical calculation, $E\left[\xi_{i k}\right]$ is shown in Table I for various values of propagation exponent and $P_{j} / P_{s}$.

\section{POWER CONTROL ERROR}

In the open-loop power control algorithm, the received signal power at the mobile is estimated by averaging the output square of a correlator [1]. The estimate at time $L T$ is given by averaging the square of (10)

$$
\begin{aligned}
\hat{S}^{2}(L)= & \frac{1}{L} \sum_{l=1}^{L} Z^{2}\left(\left.l\right|_{r_{11}, \theta_{11}}\right) \\
= & \frac{1}{L} \sum_{l=1}^{L} 2 P_{R} \xi_{11} r_{11}^{-\gamma} a_{1}^{2}(l) e^{2 \xi_{1}(l)} \\
& +\frac{2}{L} \sum_{l=1}^{L} \sqrt{2 P_{R} \xi_{11} r_{11}^{-\gamma}} a_{1}(l) e^{\xi_{1}(l)}[I(l)+J(l)] \\
& +\frac{2}{L} \sum_{l=1}^{L} I(l) J(l)+\frac{1}{L} \sum_{l=1}^{L} I^{2}(l)+\frac{1}{L} \sum_{l=1}^{L} J^{2}(l) .
\end{aligned}
$$

The mobile uses this estimate to adjust its transmit power, which is inversely proportional to the estimated power, e.g., $P_{t}=$ $1 /\left(C \cdot \hat{S}^{2}(L)\right)$, where $C$ is a weighted constant. Therefore, neglecting the loop delay on uplink, the desired signal component of the base station after despreading at the time $L T$, is given by

$$
\begin{aligned}
Z_{1}(L) & =\sqrt{\frac{2 r_{11}^{-\gamma}}{C \cdot \hat{S}^{2}(L)}} a_{1}^{\prime}(L) e^{\zeta_{1}(L)} b_{11}^{\prime}(L) \\
& =\sqrt{2 P_{\mathrm{err}}} a_{1}^{\prime}(L) b_{11}^{\prime}(L)
\end{aligned}
$$

where $a_{1}^{\prime}(L), e^{\zeta_{1}(L)}$ and $b_{11}^{\prime}(L)$ stand for the Rayleigh fading, lognormal distributed shadowing and data bit, respectively, for the uplink transmission. Notice that the shadowing terms on both up and down links are the same but the fading terms on the two links are different. $P_{\text {err }}$ is the received power of the base station, defined as

$$
P_{\mathrm{err}}=\frac{r_{11}^{-\gamma} e^{2 \zeta_{1}(L)}}{C \cdot \hat{S}^{2}(L)}
$$

Assuming the square-root of $P_{\text {err }}$ is lognormally distributed, e.g.,

$$
\sqrt{P_{\mathrm{err}}}=e^{x}
$$

one obtains $\left(1 / P_{\text {err }}\right)=e^{-2 x}$ and $\left(1 / P_{\text {err }}^{2}\right)=e^{-4 x}$. When $x$ is constant (or $x=0$ ), the received power of the base station is deterministic (perfect case). However, due to adjacent cell interference and NBI, $x$ (or $P_{\text {err }}$ ) is a random variable. Therefore, our objective here is to evaluate the mean and the standard deviation of $x$, which are shown in the Appendix B.

\section{NUMERICAL RESULTS}

The numerical results of the standard deviation (B6) of the power control error are shown for several cases. Unless noted otherwise, the following parameters are assumed: the path loss exponent $\gamma=4$, the processing gain $N=1000$, the number of active users per cell $K=20$, the number of taps of the Wiener filter $M=4$ on each side, the ratio of the interference bandwidth to the spread spectrum bandwidth $p=0.2$, the ratio of the offset of the interference carrier frequency to half of the spread 


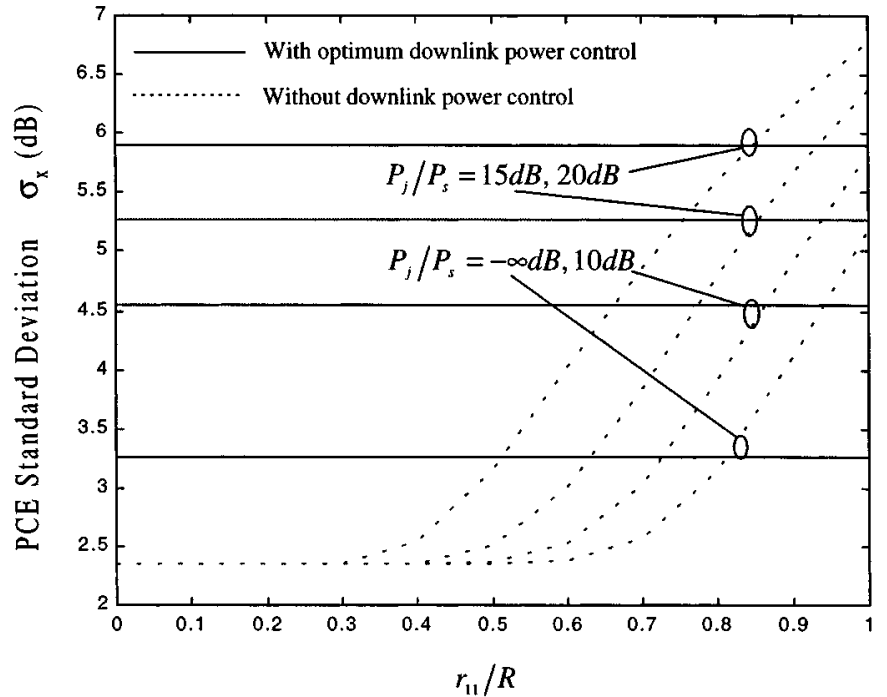

Fig. 3. Standard derivation of power control error versus relative distance.

spectrum bandwidth $q=0.2$, standard deviation of shadowing $\sigma_{\zeta}=8 \mathrm{~dB}$, the information data rate $10 \mathrm{~Kb} / \mathrm{s}$, the speed of a mobile user $v=100 \mathrm{~km} / \mathrm{h}$, the carrier frequency $f_{0}=2 \mathrm{GHz}$ (Dopplor frequency shift $f_{d}=v / \lambda=185 \mathrm{~Hz}$ ), the period for power estimation $L=60$ (or 60 bits), and the shadowing correlation distance $D=45 \mathrm{~m}$.

Fig. 3 illustrates the standard deviation of power control error when optimum downlink power control is used as a function of $r_{11} / R$ for various values of $P_{j} / P_{s}$. For comparison, the power control error without power control is also shown in the figure. Without downlink power control, assuming $\theta_{11}=\pi / 6$, a base station transmits the same power to all the users wherever they are, so that a user close to the base station will receive more power than a user far from it. Therefore, when the distance ratio is small, i.e., $r_{11} / R<0.3$, the received signal power of a mobile user is very large. However, NBI and adjacent-cell interference are relatively stable. That is, the SIR is very large. Thus, the power control error mainly results from the measurement error, which mainly depends on the measurement interval, mobile speed and shadowing correlation distance. However, when the distance ratio $\left(r_{11} / R\right)$ increases, the received signal power of a mobile decreases. That is, the SIR decreases. In this case, the power control error is caused by both interference and measurement error. Therefore, the power control error standard deviation increases as the distance ratio increases. For a given value of $P_{j} / P_{s}$, when a mobile is located at one of vertices (i.e., $r_{11} / R=1$ ), the power control error takes the maximum value, which is used for evaluation of system capacity. However, when power control is used, for a given value of $P_{j} / P_{s}$, the power control error keeps constant, irrespective of the relative distance, $r_{11} / R$. This is contrast to that without power control, where the power control error increases dramatically as $r_{11} / R$ for large values of $r_{11} / R$. It can also be seen from Fig. 3 that, in the presence of NBI, the optimum downlink power control algorithm can reduce the power control error significantly. For $P_{j} / P_{s}=-\infty, 10,15,20 \mathrm{~dB}$, the power control error is $1.9,1.3$, $1.1,0.9 \mathrm{~dB}$, respectively, less than that without power control.

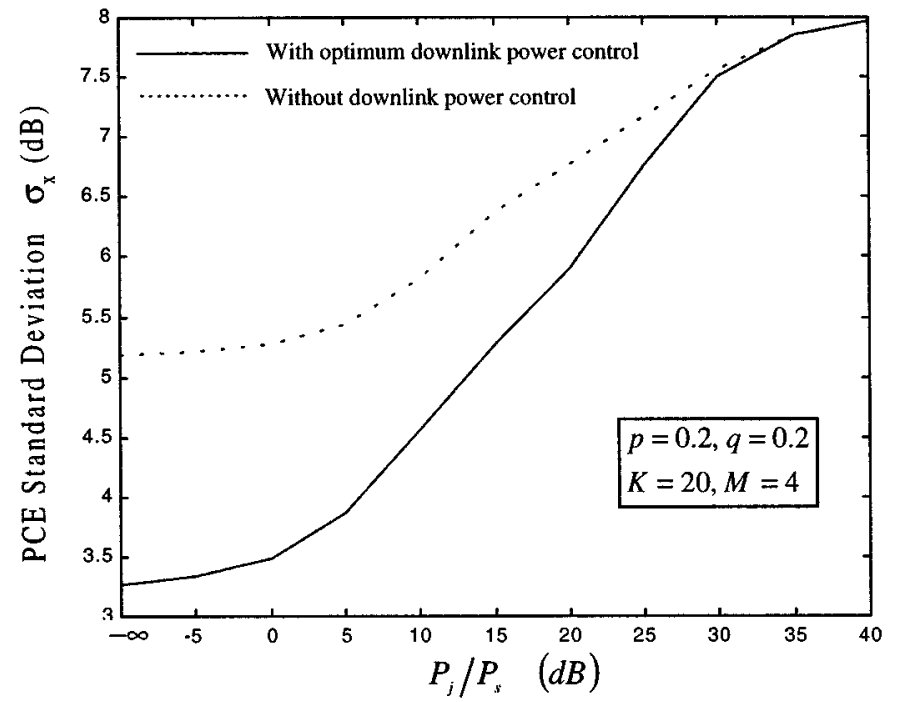

Fig. 4. Standard derivation of power control error as a function of $P_{j} / P_{s}$.

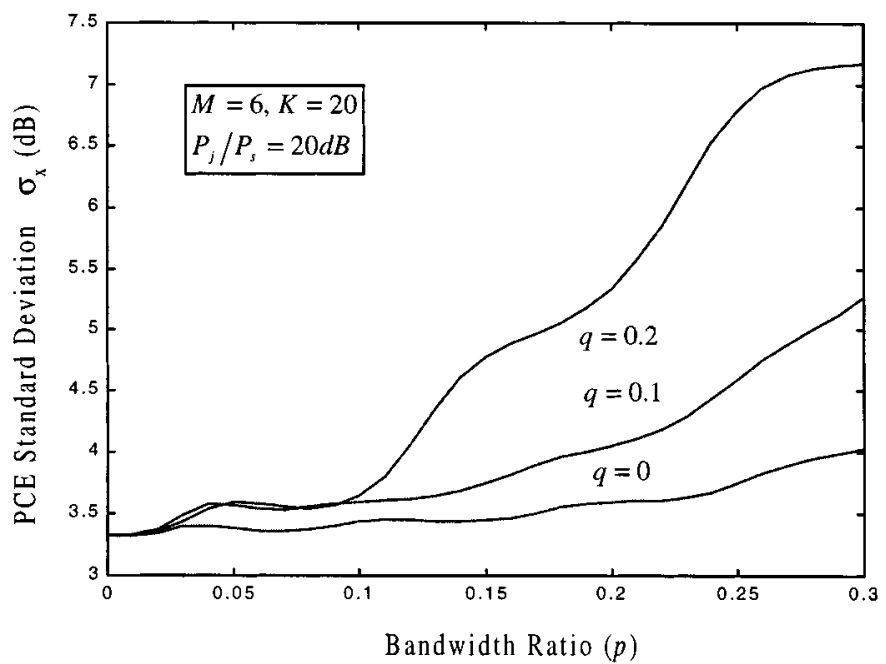

Fig. 5. Standard derivation of power control error as a function of the ratio $(p)$ of the interference BW to the spread spectrum BW.

Fig. 4 shows the power control error as a function of $P_{j} / P_{s}$ both with and without power control. It can be seen that the power control algorithm performs very well for large range of $P_{j} / P_{s}$. However, when $P_{j} / P_{s}$ is very large (i.e., $P_{j} / P_{s}>$ $30 \mathrm{~dB}$ ), the power control error for both curves approaches $8 \mathrm{~dB}$ (the maximum power control error). That is, the power control is ineffective when $P_{j} / P_{s}$ is very large.

Fig. 5 is plotted for the power control error as a function of the bandwidth ratio $(p)$ of interference bandwidth to the CDMA signal bandwidth for the offset ratio $q=0,0.1,0.2$, respectively, when power control algorithm is used. It can be seen that the power control error does not change much for small bandwidth ratio $p(p \leq 0.1)$, whereas it increases dramatically as $q$ increases for large $p(p>0.1)$.

Fig. 6 shows the power control error as a function of the offset ratio $(q)$ for different values of the number $(M)$ of each-side taps of the suppression filter. It can be seen that generally, power control error decreases as $M$ increases for a given value of $q$ and that power control error is more robust for large $M$. 


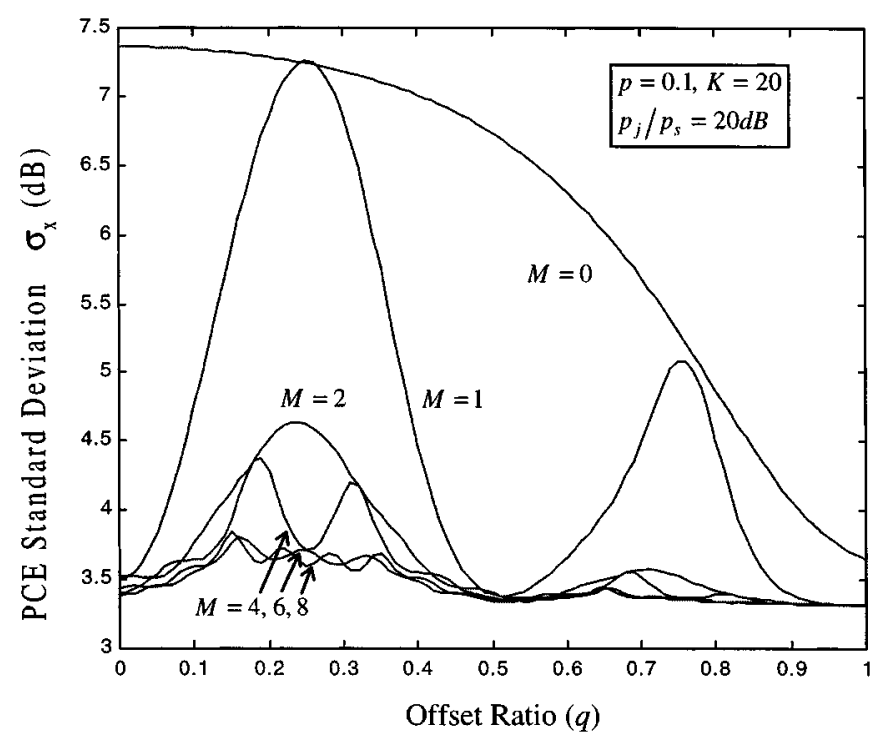

Fig. 6. Standard derivation of power control error as a function of the offset ratio $(q)$ of the interference carrier frequency to the half spread spectrum BW.

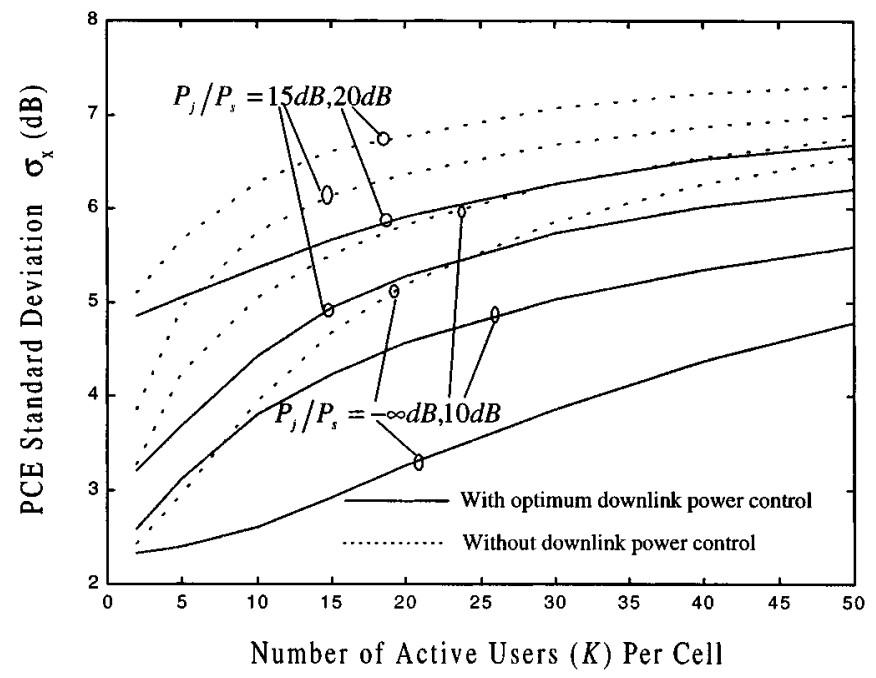

Fig. 7. Standard derivation of power control error as a function of active users $K$.

Finally, Fig. 7 illustrates the power control error as a function of the number of active users $(K)$ for various values of $P_{j} / P_{s}$. It can be seen that for a given value of power control error and given value of $P_{j} / P_{s}$, the CDMA overlay system with optimum power control can support many more users than that without power control.

\section{CONCLUSION}

The optimum open-loop power control error in cellular CDMA overlay systems has been studied. The following conclusions have been drawn:

1) For a wide range of the ratio of NBI to signal power, the optimum power control algorithm performs well.

2) In order for the power control error to be more robust against the offset ratio, a suppression filter with more taps should be used.
3) The cellular CDMA overlay system with optimum open-loop power control can support many more users than that without power control.

\section{APPENDIX A \\ DEVIATION OF THE VARIANCE OF NBI}

Equation (12) is rewritten as

$$
\begin{aligned}
J(l)= & \frac{1}{T} \sum_{m=-M}^{M} \alpha_{m} \\
& \times \int_{(l-1) T}^{l T} j\left(t-m T_{c}\right) 2 c_{11}(t) \cos \left(2 \pi f_{0} t\right) d t \\
= & \frac{1}{T} \sum_{m=-M}^{M} \alpha_{m} \int_{(l-1) T}^{l t}\left\{j_{c}\left(t-m T_{c}\right)\right. \\
& \times \cos \left[2 \pi\left(f_{0}+\Delta\right)\left(t-m T_{c}\right)\right]-j_{s}\left(t-m T_{c}\right) \\
& \left.\times \sin \left[2 \pi\left(f_{0}+\Delta\right)\left(t-m T_{c}\right)\right]\right\} \cdot 2 c_{11}(t) \cos \left(2 \pi f_{0} t\right) d t .
\end{aligned}
$$

The double-frequency component in (A1) can be removed by the integrator. Assuming $f_{0} T_{c}$ is an integer, $J(l)$ becomes

$$
\begin{aligned}
J(l)= & \frac{1}{T} \sum_{m=-M}^{M} \alpha_{m} \\
& \times \int_{(l-1) T}^{l T}\left\{j_{c}\left(t-m T_{c}\right) \cos \left[2 \pi \Delta\left(t-m T_{c}\right)\right]\right. \\
& \left.-j_{s}\left(t-m T_{c}\right) \sin \left[2 \pi \Delta\left(t-m T_{c}\right)\right]\right\} c_{11}(t) d t \\
= & \frac{1}{T} \sum_{m=-M}^{M} \alpha_{m} \int_{(l-1) T}^{l T} \hat{j}\left(t-m T_{c}\right) c_{11}(t) d t
\end{aligned}
$$

where $\hat{j}(t)$ is the low-frequency version of the NBI, $j(t)$, and is defined as

$$
\hat{j}(t)=j_{c}(t) \cos (2 \pi \Delta t)-j_{s}(t) \sin (2 \pi \Delta t) .
$$

The autocorrelation of $\hat{j}(t)$ is given by as (4), i.e., $R_{\hat{j}}(\tau)=$ $R_{j}(\tau)$, with $f_{o}$ replaced by 0 . Therefore,

$$
\begin{aligned}
J(l)= & \frac{1}{T} \sum_{m=-M}^{M} \alpha_{m} \\
& \times \int_{0}^{T} \hat{j}\left(t-(l-1) T-m T_{c}\right) c_{11}(t-(l-1) T) d t \\
= & \frac{1}{T} \sum_{m=-M}^{M} \alpha_{m} \sum_{n=1}^{N} c_{11}^{(n)} \\
& \times \int_{(n-1) T_{c}}^{n T_{c}} \hat{j}\left[t-(l-1) T-m T_{c}\right] d t \\
= & \frac{1}{T} \sum_{m=-M}^{M} \alpha_{m} \sum_{n=1}^{N} c_{11}^{(n)} \\
& \times \int_{0}^{T_{c}} \hat{j}\left[t-(l-1) T-m T_{c}-(n-1) T_{c}\right] d t \quad(\mathrm{~A} 4)
\end{aligned}
$$


where $c_{11}^{(n)}$ represents the $n$th chip of random sequence $c_{11}(t)$ and $N$ is the processing gain. The variance of $J(l)$ is given by

$$
\begin{aligned}
\sigma_{J}^{2}= & E\left[J^{2}(l)\right] \\
= & \frac{1}{T^{2}} \sum_{m_{1}=-M}^{M} \alpha_{m_{1}} \sum_{m_{2}=-M}^{M} \alpha_{m_{2}} \\
& \times \sum_{n_{1}=1}^{N} \sum_{n_{2}=1}^{N} E\left[c_{11}^{\left(n_{1}\right)} c_{11}^{\left(n_{2}\right)}\right] \\
& \cdot \int_{0}^{T_{c}} \int_{0}^{T_{c}} E\left\{\hat { j } \left[t_{1}-(l-1) T-m_{1} T_{c}\right.\right. \\
& \left.-\left(n_{1}-1\right) T_{c}\right] \hat{j}\left[t_{2}-(l-1) T-m_{2} T_{c}\right. \\
& \left.\left.-\left(n_{2}-1\right) T_{c}\right]\right\} d t_{1} d t_{2} .
\end{aligned}
$$

Since $E\left\lfloor c_{11}^{\left(n_{1}\right)} c_{11}^{\left(n_{2}\right)}\right\rfloor=1$ and 0 for $n_{1}=n_{2}$ and $n_{1} \neq n_{2}$, respectively, $\sigma_{J}^{2}$ reduces to

$$
\begin{aligned}
\sigma_{J}^{2}= & \frac{1}{T^{2}} \sum_{m_{1}=-M}^{M} \alpha_{m_{1}} \sum_{m_{2}=-M}^{M} \alpha_{m_{2}} \\
& \times \sum_{n=1}^{N} \int_{-T_{c}}^{T_{c}} R_{\hat{j}}\left[\tau-\left(m_{1}-m_{2}\right) T_{c}\right]\left(T_{c}-|\tau|\right) d \tau \\
= & \frac{N T_{c}^{2}}{T^{2}} \sum_{m_{1}=-M}^{M} \alpha_{m_{1}} \sum_{m_{2}=-M}^{M} \alpha_{m_{2}} \\
& \times \int_{-1}^{1} R_{j}\left\{\left[\tau-\left(m_{1}-m_{2}\right)\right] T_{c}\right\}(1-|\tau|) d \tau \\
= & \frac{P_{j}}{N} \sum_{m_{1}=-M}^{M} \alpha_{m_{1}} \sum_{m_{2}=-M}^{M} \alpha_{m_{2}} \\
& \times\left[Q\left(m_{1}, m_{2}\right)+Q\left(-m_{1},-m_{2}\right)\right]
\end{aligned}
$$

where

$$
\begin{aligned}
Q\left(m_{1}, m_{2}\right)= & \int_{0}^{1}(1-|\tau|) \operatorname{sinc}\left\{2 \pi\left[\tau-\left(m_{1}-m_{2}\right)\right] p\right\} \\
& \times \cos \left\{2 \pi\left[\tau-\left(m_{1}-m_{2}\right)\right] q\right\} d \tau
\end{aligned}
$$

\section{APPENDIX B}

DERIVATION OF THE MEAN AND STANDARD DEVIATION OF POWER CONTROL ERROR

From (28)-(31), one obtains

$$
\begin{aligned}
\frac{1}{P_{\mathrm{err}}}= & e^{-2 x} \\
= & \frac{C}{L} \sum_{l=1}^{L} 2 P_{R} \xi_{11} a_{1}^{2}(l) e^{2 \xi_{1}(l)-2 \zeta_{1}(L)}+\frac{2 C}{L r_{11}^{-\gamma / 2}} \\
& \times \sum_{l=1}^{L} \sqrt{2 P_{R} \xi_{11}} a_{1}(l) e^{\xi_{1}(l)-2 \zeta_{1}(L)}[I(l)+J(l)] \\
& +\frac{2 C}{L r_{11}^{-\gamma}} \sum_{l=1}^{L} e^{-2 \zeta_{1}(L)} I(l) J(l) \\
& +\frac{C}{L r_{11}^{-\gamma}} \sum_{l=1}^{L} e^{-2 \zeta_{1}(L)}\left[I^{2}(l)+J^{2}(l)\right]
\end{aligned}
$$

and

$$
\begin{aligned}
\frac{1}{P_{\mathrm{err}}^{2}}= & e^{-4 x} \\
= & \frac{4 C^{2} P_{R}^{2} \xi_{11}^{2}}{L^{2}} \sum_{l_{1}=1}^{L} \sum_{l_{2}=1}^{L} a_{1}^{2}\left(l_{1}\right) a_{1}^{2}\left(l_{2}\right) \\
& \times e^{2 \zeta_{1}\left(l_{1}\right)+2 \zeta_{1}\left(l_{2}\right)-4 \zeta_{1}(L)}+\frac{8 C^{2} P_{R} \xi_{11}}{L^{2} r_{11}^{-\gamma}} \\
& \times \sum_{l_{1}=1}^{L} \sum_{l_{2}=1}^{L} a_{1}\left(l_{1}\right) a_{1}\left(l_{2}\right) e^{\zeta_{1}\left(l_{1}\right)+\zeta_{1}\left(l_{2}\right)-4 \zeta_{1}(L)} \\
& \times\left[I\left(l_{1}\right) I\left(l_{2}\right)+J\left(l_{1}\right) J\left(l_{2}\right)\right] \\
& +\frac{4 C^{2}}{L^{2} r_{11}^{-2 \gamma}} \sum_{l_{1}=1}^{L} \sum_{l_{2}=1}^{L} e^{-4 \zeta_{1}(L)} I\left(l_{1}\right) I\left(l_{2}\right) J\left(l_{1}\right) J\left(l_{2}\right) \\
& +\frac{C^{2}}{L^{2} r_{11}^{-2 \gamma}} \sum_{l_{1}=1}^{L} \sum_{l_{2}=1}^{L} e^{-4 \zeta_{1}(L)}\left[I^{2}\left(l_{1}\right) I^{2}\left(l_{2}\right)\right. \\
& \left.+2 I^{2}\left(l_{1}\right) J^{2}\left(l_{2}\right)+J^{2}\left(l_{1}\right) J^{2}\left(l_{2}\right)\right] \\
& +\frac{4 C^{2} P_{R} \xi_{11}}{L^{2} r_{11}^{-\gamma}} \sum_{l_{1}=1}^{L} \sum_{l_{2}=1}^{L} a_{1}^{2}\left(l_{1}\right) e^{2 \zeta_{1}\left(l_{1}\right)-4 \zeta_{1}(L)} \\
& \times\left[I^{2}\left(l_{2}\right)+J^{2}\left(l_{2}\right)\right] . \\
& \mathrm{B} 2) \\
&
\end{aligned}
$$

Since $x$ is Gaussian distributed, $E\left\lfloor e^{x}\right\rfloor=\exp \left(\bar{x}+\sigma_{x}^{2} / 2\right)$, where $\bar{x}$ and $\sigma_{x}$ stand for the mean and standard deviation of power control error. In the following, it is shown how to obtain $\bar{x}$ and $\sigma_{x}$.

From (B1) and (B2), we have

$$
\begin{aligned}
& E\left[e^{-2 x}\right]=e^{-2 \bar{x}+2 \sigma_{x}^{2}}=C U, \\
& E\left[e^{-4 x}\right]=e^{-4 \bar{x}+8 \sigma_{x}^{2}}=C^{2} V
\end{aligned}
$$

where $U$ and $V$ will be derived later. From (B3) and (B4), $\bar{x}$ and $\sigma_{x}$ can be derived, i.e.,

$$
\begin{aligned}
\bar{x} & =\frac{1}{2} \ln \left(\sqrt{V} / C U^{2}\right), \\
\sigma_{x} & =\frac{1}{2} \sqrt{\ln \left(V / U^{2}\right)} .
\end{aligned}
$$

By choosing $C$ appropriately (i.e., $C=\sqrt{V} / U^{2}$ ), the mean of the power control error can be set to zero $(\bar{x}=0)$, corresponding to an unbiased estimate.

Since all $a_{1}(l), e^{\zeta_{1}(l)}, I(l)$ and $J(l)$ are independent, $E[I(l)]=E[J(l)]=0$, and $E\left[I\left(l_{1}\right) I\left(l_{2}\right)\right]=E\left[J\left(l_{1}\right) J\left(l_{2}\right)\right]=$ 0 , for $l_{1} \neq l_{2}$, the cross-terms with odd-orders of $I(l)$ and $J(l)$ have zero expectation. Thus, from (B1) and (B3), we have

$$
\begin{aligned}
U= & \frac{2 P_{R} \xi_{11}}{L} \sum_{l=1}^{L} E\left[a_{1}^{2}(l) e^{2 \zeta_{1}(l)-2 \zeta_{1}(L)}\right] \\
& +\frac{1}{L r_{11}^{-\gamma}} \sum_{l=1}^{L} E\left[e^{-2 \zeta_{1}(L)}\left(I^{2}(l)+J^{2}(l)\right)\right] \\
= & \frac{2 P_{R} \xi_{11}}{L} \sum_{l=1}^{L} E\left[a_{1}^{2}(l)\right] E\left[e^{2 \zeta_{1}(l)-2 \zeta_{1}(L)}\right]+\frac{1}{L r_{11}^{-\gamma}}
\end{aligned}
$$




$$
\begin{aligned}
& +\sum_{l=1}^{L} E\left[e^{-2 \zeta_{1}(L)}\right] E\left[I^{2}(l)+J^{2}(l)\right] \\
= & \frac{2 P_{R} \xi_{11}}{L} \sum_{l=1}^{L} \exp \left(4 \sigma_{\zeta_{1}}^{2}-4 C_{\zeta_{1}}((l-L) T)\right) \\
& +\frac{1}{r_{11}^{-\gamma}} e^{2 \sigma_{\xi_{1}}^{2}}\left(\sigma_{I}^{2}+\sigma_{J}^{2}\right) \\
= & \frac{P_{s} \xi_{11}}{L R^{-\gamma}} \sum_{l=1}^{L} \exp \left(2 \sigma_{\zeta_{1}}^{2}-4 C_{\zeta_{1}}((l-L) T)\right) \\
& +\frac{1}{r_{11}^{-\gamma}} e^{2 \sigma_{\zeta_{1}}^{2}}\left(\sigma_{I}^{2}+\sigma_{J}^{2}\right)
\end{aligned}
$$

where $E\left\lfloor a_{1}^{2}(l)\right\rfloor=1, P_{s}$ is given by $(20)$ and $C_{\zeta_{1}}(\tau)$ is the autocovariance function of $\zeta(t)$, given by [8]

$$
\begin{aligned}
C_{\zeta_{1}}(\tau) & =E\left[\zeta_{1}(t) \zeta_{1}(t+\tau)\right]-E^{2}\left[\zeta_{1}(t)\right] \\
& =\sigma_{\zeta_{1}}^{2} e^{-v|\tau| / D}
\end{aligned}
$$

where $v$ stands for the speed of a mobile, $D$ represents shadowing correlation distance and has been measured as hundreds of meters for conventional terrestrial cells, and tens of meters for terrestrial microcells.

From (B2) and (B4), we have

$$
\begin{aligned}
V= & \frac{4 P_{R}^{2} \xi_{11}^{2}}{L^{2}} \sum_{l_{1}=1}^{L} \sum_{l_{2}=1}^{L} E\left[a_{1}^{2}\left(l_{1}\right) a_{1}^{2}\left(l_{2}\right)\right. \\
& \times e^{\left.2 \zeta_{1}\left(l_{1}\right)+2 \zeta_{1}\left(l_{2}\right)-4 \zeta_{1}(L)\right]} \\
& +\frac{8 P_{R} \xi_{11}}{L^{2} r_{11}^{-\gamma}} \sum_{l_{1}=1}^{L} \sum_{l_{2}=1}^{L} E\left\{a_{1}\left(l_{1}\right) a_{1}\left(l_{2}\right)\right. \\
& \left.\times e^{\zeta_{1}\left(l_{1}\right)+\zeta_{1}\left(l_{2}\right)-4 \zeta_{1}(L)}\left[I\left(l_{1}\right) I\left(l_{2}\right)+J\left(l_{1}\right) J\left(l_{2}\right)\right]\right\} \\
& +\frac{4}{L^{2} r_{11}^{-2 \gamma}} \sum_{l_{1}=1}^{L} \sum_{l_{2}=1}^{L} E\left[e^{-4 \zeta_{1}(L)} I\left(l_{1}\right) I\left(l_{2}\right) J\left(l_{1}\right) J\left(l_{2}\right)\right] \\
& +\frac{1}{L^{2} r_{11}^{-2 \gamma}} \sum_{l_{1}=1}^{L} \sum_{l_{2}=1}^{L} E\left\{e ^ { - 4 \zeta _ { 1 } ( L ) } \left[I^{2}\left(l_{1}\right) I^{2}\left(l_{2}\right)\right.\right. \\
& \left.\left.+2 I^{2}\left(l_{1}\right) J^{2}\left(l_{2}\right)+J^{2}\left(l_{1}\right) J^{2}\left(l_{2}\right)\right]\right\} \\
& +\frac{4 P_{R} \xi_{11}}{L^{2} r_{11}^{-\gamma}} \sum_{l_{1}=1}^{L} \sum_{l_{2}=1}^{L} E\left\{a_{1}^{2}\left(l_{1}\right) e^{2 \zeta_{1}\left(l_{1}\right)-4 \zeta_{1}(L)}\right. \\
& \left.\times\left[I^{2}\left(l_{2}\right)+J^{2}\left(l_{2}\right)\right]\right\} \\
= & \frac{4 P_{R}^{2} \xi_{11}^{2}}{L^{2}} \sum_{l_{1}=1}^{L} \sum_{l_{2}=1}^{L} E\left[a_{1}^{2}\left(l_{1}\right) a_{1}^{2}\left(l_{2}\right)\right] E \\
& \times\left[e^{2 \zeta_{1}\left(l_{1}\right)+2 \zeta_{1}\left(l_{2}\right)-4 \zeta_{1}(L)}\right] \\
& +\frac{8 P_{R} \xi_{11}}{L^{2} r_{11}^{-\gamma}} \sum_{l=1}^{L} E\left[e^{2 \zeta_{1}(l)-4 \zeta_{1}(L)}\right] E\left[I^{2}(l)+J^{2}(l)\right] \\
& +\frac{4}{L^{2} r_{11}^{-2 \gamma}} \sum_{l=1}^{L} E\left[e^{-4 \xi_{1}(L)}\right] E\left[I^{2}(l)\right] E\left[J^{2}(l)\right] \\
&
\end{aligned}
$$

$$
\begin{aligned}
& +\frac{1}{L^{2} r_{11}^{-2 \gamma}} \sum_{l_{1}=1}^{L} \sum_{l_{2}=1}^{L} E\left[e^{-4 \zeta_{1}(L)}\right] E\left[I^{2}\left(l_{1}\right) I^{2}\left(l_{2}\right)\right. \\
& \left.+2 I^{2}\left(l_{1}\right) J^{2}\left(l_{2}\right)+J^{2}\left(l_{1}\right) J^{2}\left(l_{2}\right)\right] \\
& +\frac{4 P_{R} \xi_{11}}{L r_{11}^{-\gamma}} \sum_{l=1}^{L} E\left[e^{2 \zeta_{1}(l)-4 \zeta_{1}(L)}\right] E\left[I^{2}(l)+J^{2}(l)\right]
\end{aligned}
$$

where $E\left\lfloor a_{1}^{2}\left(l_{1}\right) a_{1}^{2}\left(l_{2}\right)\right\rfloor$ is the autocorrelation function of $a_{1}^{2}(l)$, defined as

$$
\begin{aligned}
R_{a_{1}^{2}}\left(l_{2}-l_{1}\right) & =E\left\lfloor a_{1}^{2}\left(l_{1}\right) a_{1}^{2}\left(l_{2}\right)\right\rfloor \\
& =1+\rho^{2}\left(l_{2}-l_{1}\right)
\end{aligned}
$$

where $\rho\left(l_{2}-l_{1}\right)$ is the normalized autocovariance function of the Rayleigh process, given by [9]

$$
\rho\left(l_{2}-l_{1}\right)=J_{0}\left(2 \pi f_{d}\left|\left(l_{2}-l_{1}\right) T\right|\right)
$$

where $f_{d}$ is a maximum Doppler frequency or fading rate, given by $f_{d}=v / \lambda$, where $\lambda$ is a carrier wavelength. $J_{0}(x)$ is a Bessel function of the first kind of zeroth order.

Since both $I(l)$ and $J(l)$ are Gaussian, we have

$$
E\left[I^{2}\left(l_{1}\right) I^{2}\left(l_{2}\right)\right]= \begin{cases}3 \sigma_{I}^{4}, & l_{1}=l_{2} \\ \sigma_{I}^{4}, & l_{1} \neq l_{2}\end{cases}
$$

and

$$
E\left[J_{1}^{2}\left(l_{1}\right) J_{1}^{2}\left(l_{2}\right)\right]=\left\{\begin{array}{ll}
3 \sigma_{J}^{4}, & l_{1}=l_{2} \\
\sigma_{J}^{4}, & l_{1} \neq l_{2}
\end{array} .\right.
$$

Thus,

$$
\begin{aligned}
& \sum_{l_{1}=1}^{L} \sum_{l_{2}=1}^{L} E\left[I^{2}\left(l_{1}\right) I^{2}\left(l_{2}\right)\right] \\
& \quad=\sum_{l_{1}=1}^{L}\left\{E\left[I^{4}(l)\right]+\sum_{\substack{l_{2}=1 \\
l_{2} \neq l_{1}}}^{L} E\left[I^{2}\left(l_{1}\right) I^{2}\left(l_{2}\right)\right]\right\} \\
& \quad=3 L \sigma_{I}^{4}+\left(L^{2}-L\right) \sigma_{I}^{4}
\end{aligned}
$$

and

$$
\sum_{l_{1}=1}^{L} \sum_{l_{2}=1}^{L} E\left[J_{1}^{2}\left(l_{1}\right) J_{1}^{2}\left(l_{2}\right)\right]=3 L \sigma_{J}^{4}+\left(L^{2}-L\right) \sigma_{J}^{4}(\mathrm{~B}
$$

$V$ in (B9) can be simplified to

$$
\begin{aligned}
V= & \frac{4 P_{R}^{2} \xi_{11}^{2}}{L^{2}} \sum_{l_{1}=1}^{L} \sum_{l_{2}=1}^{L}\left[1+\rho^{2}\left(l_{1}-l_{2}\right)\right] \\
& \cdot \exp \left\{12 \sigma_{\zeta_{1}}^{2}+4 C_{\zeta_{1}}\left[\left(l_{1}-l_{2}\right) T\right]\right. \\
& \left.-8 C_{\zeta_{1}}\left[\left(l_{1}-L\right) T\right]-8 C_{\zeta_{1}}\left[\left(l_{2}-L\right) T\right]\right\} \\
& +\frac{8 P_{R} \xi_{11}}{L^{2} r_{11}^{-\gamma}\left(\sigma_{I}^{2}+\sigma_{J}^{2}\right)} \\
& \times \sum_{l=1}^{L} \exp \left\{10 \sigma_{\zeta_{1}}^{2}-8 C_{\zeta_{1}}[(l-L) T]\right\} \\
& +\frac{4}{L r_{11}^{-2 \gamma}} \sigma_{I}^{2} \sigma_{J}^{2} e^{8 \sigma_{\zeta_{1}}^{2}}+\left(1+\frac{2}{L}\right) \frac{\left(\sigma_{I}^{4}+\sigma_{J}^{4}\right)}{r_{11}^{-2 \gamma}} e^{8 \sigma_{\zeta_{1}}^{2}} \\
& +\frac{2}{r_{11}^{-2 \gamma}} \sigma_{1}^{2} \sigma_{J}^{2} e^{8 \sigma_{\zeta_{1}}^{2}}+\frac{4 P_{R} \xi_{11}}{L r_{11}^{-\gamma}}\left(\sigma_{I}^{2}+\sigma_{J}^{2}\right)
\end{aligned}
$$




$$
\begin{aligned}
& \times \sum_{l=1}^{L} \exp \left\{10 \sigma_{\zeta_{1}}^{2}-8 C_{\zeta_{1}}[(l-L) T]\right\} \\
= & \frac{P_{s}^{2} \xi_{11}^{2}}{L^{2} R^{-2 \gamma}} \sum_{l_{1}=1}^{L} \sum_{l_{2}=1}^{L}\left[1+\rho^{2}\left(l_{1}-l_{2}\right)\right] \\
& \cdot \exp \left\{8 \sigma_{\zeta_{1}}^{2}+4 C_{\zeta_{1}}\left[\left(l_{1}-l_{2}\right) T\right]-8 C_{\zeta_{1}}\left[\left(l_{1}-L\right) T\right]\right. \\
& \left.-8 C_{\zeta_{1}}\left[\left(l_{2}-L\right) T\right]\right\} \\
& +\frac{2 P_{s} \xi_{11}}{L r_{11}^{-\gamma} R-\gamma}\left(1+\frac{2}{L}\right)\left(\sigma_{I}^{2}+\sigma_{J}^{2}\right) \\
& \times \sum_{l=1}^{L} \exp \left\{8 \sigma_{\zeta_{1}}^{2}-8 C_{\zeta_{1}}[(l-L) T]\right\} \\
& +\frac{2}{r_{11}^{-2 \gamma}}\left(1+\frac{2}{L}\right) \sigma_{I}^{2} \sigma_{J}^{2} e^{8 \sigma_{\zeta_{1}}^{2}} \\
& +\frac{1}{r_{11}^{-2 \gamma}}\left(1+\frac{2}{L}\right)\left(\sigma_{I}^{4}+\sigma_{J}^{4}\right) e^{8 \sigma_{\zeta_{1}}^{2}} .
\end{aligned}
$$

\section{REFERENCES}

[1] A. M. Monk and L. B. Milstein, "Open-loop power control error in a land mobile satellite system," IEEE J. Select. Areas Commun., vol. 13, pp. 205-212, Feb. 1995.

[2] A. J. Goldsmith, L. J. Greenstein, and G. J. Foschini, "Error statistics of real-time power measurements in cellular channels with multipath and shadowing," IEEE Trans. Veh. Technol., vol. 43, pp. 439-446, Aug. 1994.

[3] W. C. Y. Lee, "Overview of cellular CDMA," IEEE Trans. Veh. Technol., vol. 40, pp. 291-302, May 1991.

[4] R. R. Gejji, "Forward-link-power control in CDMA cellular systems," IEEE Trans. Veh. Technol., vol. 41, pp. 532-536, Nov. 1992.

[5] J. Wang and L. B. Milstein, "CDMA overlay situations for microcellular mobile communications," IEEE Trans. Commun., vol. 43, pp. 603-614, Feb. 1995.

[6] L. A. Rusch and H. V. Poor, "Narrowband interference suppression in CDMA spread spectrum communications," IEEE Trans. Commun., vol. 42, pp. 1969-1979, Feb. 1994.

[7] M. B. Pursley, "Performance evaluation for phase-coded spread spectrum multiple access communications, part I: System analysis," IEEE Trans. Commun., vol. 25, pp. 295-299, Aug. 1977.
[8] M. Gudmundson, "Correlation model for shadow fading in mobile radio systems," Electron. Lett., vol. 27, pp. 2145-2146, Nov. 1991.

[9] R. H. Clarke, "A statistical theory of mobile radio reception," Bell Syst. Tech. J., vol. 47, pp. 957-1000, July 1968

Jiangzhou Wang (M'91-SM'94) received the B.S. and M.S. degrees in electrical engineering from Xidian University, Xian, China, in 1983 and 1985, respectively, and the Ph.D. degree (with greatest distinction) in electrical engineering from the University of Ghent, Belgium, in 1990.

From 1990 to 1992, he was a Postdoctoral Fellow with the University of California, San Diego, where he worked on the research and development of cellular CDMA systems. From 1992 to 1995, he was a Senior System Engineer with Rockwell International Corporation, Newport Beach, CA, where he worked on the development and system design of wireless communications. Since 1995, he has been with the University of Hong Kong, where he is currently an Associate Professor. He has held a Visiting Professor position in NTT DoCoMo, Japan. He has published over 100 papers, including more than 20 IEEE transactions and journal papers in the areas of wireless mobile and spread spectrum communications. He has written and edited two books, entitled Broadband Wireless Communications (Kluwer: 2001) and "3G Mobile Enhanced Technologies" (Norwell, MA: Artech House, 2001), respectively.

Dr. Wang was a Technical Chairman of IEEE Workshop in 3G Mobile Communications, 2000. He is an Editor for IEEE TRANSACTIONS ON COMMUNICATIONS and a Guest Editor for IEEE JOURNAL ON SELECTED AREAS In Communications. He holds one U.S. patent in the GSM system. He is listed in Who's Who in the World.

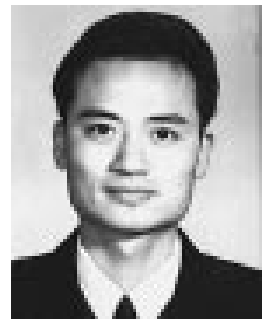

Ai Yu was born in 1968. He received the M.Sc. degree in electronic engineering, from Nanjing University of Science and Technology, P.R. China, in 1995, and the Ph.D. degree in electrical engineering from the University of Hong Kong, in 2000. During his graduate studies, he did research on spread spectrum and code division multiple access.

From 1988 to 1992, he was an Electrical Engineer with Yanzhou Marine Electronic Apparatus Institute, P.R. China, where his duties were the analysis and design of marine communication systems. Currently, he is with McGill University, Montreal, QC, Canada, as a Postdoctoral Fellow in wireless communication systems. 